Pneumonia leading to respiratory failure is a common cause of admission to medical and intensive care units worldwide and associated with significant morbidity and mortality, particularly when diagnosis is delayed. Diagnosis can be challenging and existing tools (clinical examination, CXR or CT) have their recognised flaws. TUS may be an alternative solution, offering patients a bedside investigation that provides clinicians with instant feedback to inform treatment decisions.

We searched MEDLINE, EMBASE and the Science Citation Index Expanded (inception to October 2013) for studies relating to the diagnostic use of TUS in adults with acute respiratory failure due to radiographic consolidation, focusing on studies using CT as their reference standard. Two reviewers independently extracted data from eligible studies and assessed study quality using QUADAS-2.

Results Three cohort studies, all based in an ICU setting, with a total of 134 participants met inclusion criteria. Two studies were at high risk of potential bias, whilst the third had limitations of applicability. The reported sensitivity $(0.91$ to 1.00$)$ and specificity $(0.78-1.00)$ of TUS in expert hands for CT-detected consolidation was superior to that for CXR (sensitivity 0.38 and 0.68 ; specificity 0.89 and 0.95 ). Outside the inclusion criteria, a number of studies of patients with consolidation but no respiratory failure also suggested TUS might have greater diagnostic sensitivity than CXR.

Conclusion TUS remains, at present, a technology with limited evidence to support a front-line role in the assessment of patients with respiratory failure to detect lung consolidation. However, the evidence available is promising and well-designed clinical studies are necessary to ascertain whether TUS can influence relevant outcomes for patient benefit.

\section{S42 SAFETY AND ACCEPTABILITY OF BEDSIDE ULTRASOUND-GUIDED TRANSTHORACIC LUNG NEEDLE ASPIRATION (TLNA) IN PNEUMONIA}

JM Wrightson, DWM Crook, NM Rahman, SJ Chapman. University of Oxford, Oxford, UK

\subsection{6/thoraxjnl-2014-206260.48}

Given the increasing importance of establishing a microbiological aetiology in pneumonia, we undertook a study assessing the safety and acceptability of bedside ultrasound-guided TLNA (REC No. 09/H0605/12). TLNA has previously reported to have been predominantly undertaken without radiological control.

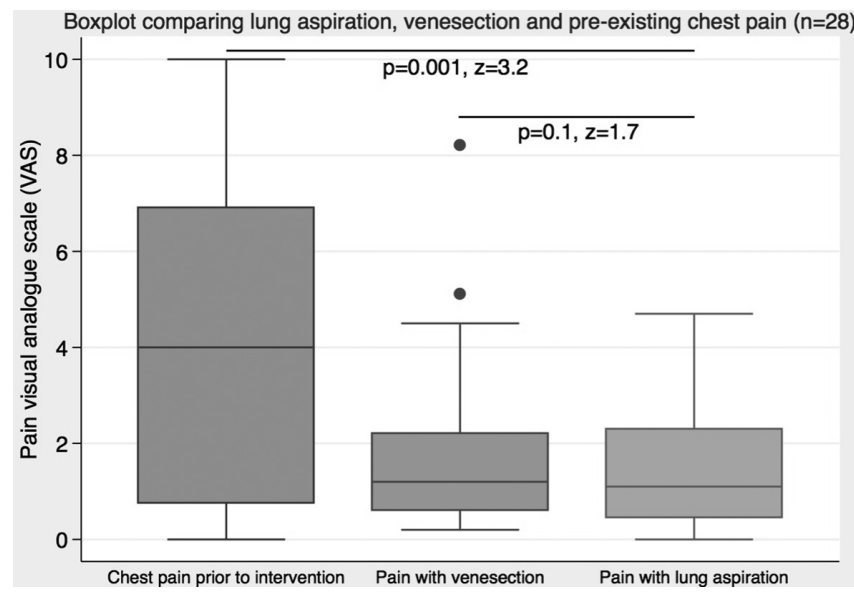

Abstract S42 Figure 1
Methods Participants with community- or hospital-acquired pneumonia completed a baseline assessment of chest pain and pain associated with phlebotomy using a $10 \mathrm{~cm}$ visual analogue scale (VAS). Post procedure, participants assessed pain associated with TLNA, and undertook a Likert-based evaluation of the procedure.

Up to $3 \mathrm{mg} / \mathrm{kg}$ lidocaine was used to anaesthetise the skin and pleura. An ultrafine $25 \mathrm{G}$ needle, attached to a $20 \mathrm{ml}$ luer lock syringe containing $3.5 \mathrm{ml} 0.9 \%$ sodium chloride solution was inserted into consolidated lung under direct ultrasound guidance by a Respiratory Physician. $0.5 \mathrm{ml}$ of the sodium chloride was injected followed by aspiration with gentle agitation $(3 \mathrm{~mL}$ of sodium chloride remaining in the syringe as a carrier solution). The needle was then withdrawn. Any pleural fluid present was also aspirated separately.

Samples underwent culture and 16S rRNA gene analysis.

All participants had follow-up chest X-rays post procedure to evaluate for pneumothorax. Participants were systematically assessed while inpatient, and again at 30 days, to assess for any adverse events.

Results 28 participants underwent TLNA, 27 using ultrasound (and one using CT-guidance). No patients experienced haemoptysis or pneumothorax. All patients either 'strongly agreed' (most commonly) or 'agreed' with the statements: 'The lung fluid sample to diagnose your pneumonia was tolerable'; and 'I would have the lung fluid sample again if my doctors thought it was essential'. The VAS-assessed pain of TLNA was significantly lower than any pre-existing chest pain, being similar to any pain associated with venesection (see Figure).

At day 30, one patient had mild ongoing pain at the site of both TLNA and subsequent chest tube insertion, although the relative contribution of each procedure to this pain was unclear.

TLNA increased culture or sequencing-based aetiological diagnosis from $3 / 28$ to $14 / 28$ (18/28 when including pleural fluid analysis).

Conclusions Physician-performed bedside ultrasound-guided TLNA appears safe and well-tolerated.

\section{S43 REAL WORLD EXPERIENCE OF THE USE OF PET-CT FOR DISTINGUISHING BETWEEN BENIGN PLEURAL DISEASE AND MALIGNANT MESOTHELIOMA}

K Prior, J Fingleton, T Howell. Plymouth Hospitals NHS Trust, Plymouth, UK

10.1136/thoraxjnl-2014-206260.49

Patients presenting with pleural disease on a background of asbestos exposure pose a diagnostic dilemma. Malignant mesothelioma and benign pleural disease have similar radiological appearances but markedly different prognoses. Definitive histological diagnosis is gold standard, however, there are small case series where PET-CT has been compared to pleural biopsy. These have suggested cut-off standardised uptake values (SUV) of 2.0-3.0, with reported sensitivity of $94.1-100 \%$ and specificity of $94-100 \%$ for excluding pleural malignancy.

It has been suggested that where the CT appearances are more in keeping with a benign aetiology, pleural avidity on PET-CT may be able to adequately distinguish between benign and malignant disease, identifying a low-risk population that can be observed in preference to proceeding to thoracoscopy.

We are a cardiothoracic centre which utilises PET-CT in this way. We aimed to review our single-centre experience to see if our outcomes were consistent with the reported data. 
All PET-CT reports carried out since 2007 with the mention of pleura/pleural within the request or report were reviewed. Radiological reports and patient records were examined, scans requested primarily for assessment of the pleura were included. Indication, radiological diagnosis, final diagnosis, presence of histological confirmation and duration of follow-up were determined. All patients with at least 6 months follow-up were analysed.

185 PET CT scan reports were reviewed, of which 28 were carried out primarily for assessment of pleural disease. 9 were found to have high SUVs suggestive of malignancy. 7 of which were demonstrated to be mesothelioma, 1 pleural tumour, 1 recurrence of non-small cell lung cancer. The remaining 19 were reported to have low SUVs, consistent with benign pleural disease.

For those with PET findings consistent with benign pleural disease, follow up data was available for a median of 12 months (Min 6- Max 66). One patient underwent pleural biopsy, which was consistent with benign disease. None of those designated as benign pleural disease based on PET-CT appearances were subsequently found to have pleural malignancy.

Our findings are consistent with previously published data and support the utility of PET-CT scanning in differentiating benign from malignant pleural disease in a clinical setting.
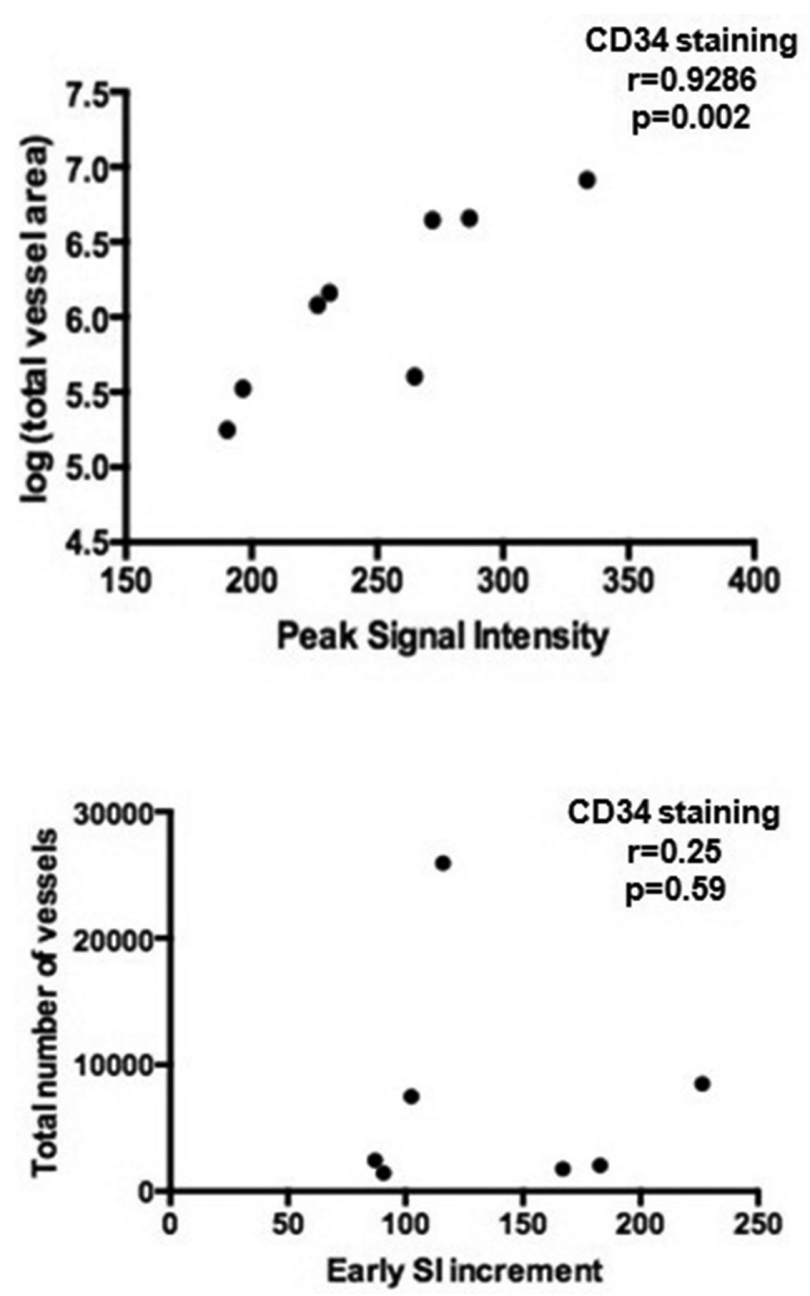

544

EARLY CONTRAST KINETICS DURING MAGNETIC RESONANCE IMAGING IN PATIENTS WITH SUSPECTED MESOTHELIOMA

${ }^{1} S$ Tsim, ${ }^{2} \mathrm{CA}$ Humphries, ${ }^{3} \mathrm{DB}$ Stobo, ${ }^{4} \mathrm{JE}$ Foster, ${ }^{4} \mathrm{R}$ Woodward, ${ }^{2} \mathrm{C}$ Dick, ${ }^{1} \mathrm{KG}$ Blyth. ${ }^{1}$ Department of Respiratory Medicine, Southern General Hospital, Glasgow, UK; ${ }^{2}$ Department of Pathology, Southern General Hospital, Glasgow, UK; ${ }^{3}$ Department of Radiology, Southern General Hospital, Glasgow, UK; ${ }^{4}$ Glasgow Clinical Imaging Research Facility, Glasgow, UK

\subsection{6/thoraxjnl-2014-206260.50}

Introduction and objectives The biology of Malignant Pleural Mesothelioma (MPM) is poorly understood, reflected in inexplicable heterogeneity in survival and therapeutic responses. Tumour angiogenesis has been identified as a therapeutic target and high tumour vascularity-to-stroma ratio is a poor prognosis marker. We report preliminary results of a pilot study conducted to establish and validate dynamic contrast-enhanced (CE) magnetic resonance imaging (MRI) methodology for the non-invasive assessment of MPM tumour vascularity.

Methods 15 patients with suspected MPM were recruited prospectively. All had Pleural MRI (3T Siemens) 3-5 days prior to Medical Thoracoscopy (MT). Imaging protocols were developed utilising patients 1-6. In the remaining 9, T1-weighted VIBE images were acquired (single isotropic volume in the coronal plane) at baseline and $4.5,9$ and 13.5 min post-injection of Gadolinium contrast $(0.1 \mathrm{mmol} / \mathrm{kg})$. Signal Intensity (SI) was measured within 15 regions of interest containing representative
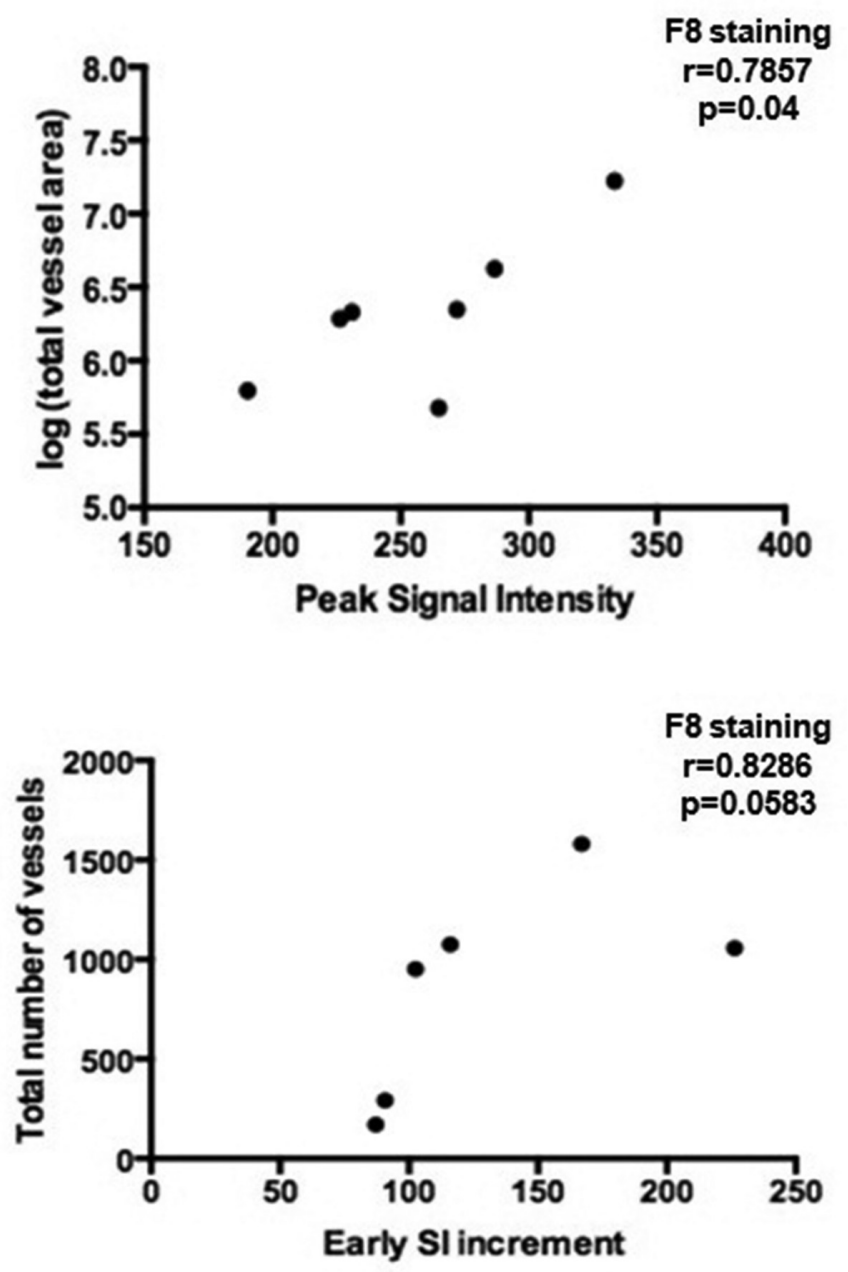

Abstract S44 Figure 1 Relationships between contrast kinetic parameters and tissue vascularity 